\title{
In vitro Bioassay of Allelopathic Activities of a Mangrove Tree, Kandelia obovata, and Fast-growing Trees, Betula platyphylla and Populus alba, Using Protoplast Co-culture Method
}

\author{
Hamako Sasamoto $^{1,2} \&$ Shinso Yokota $^{3}$ \\ ${ }^{1}$ Faculty of Environment and Information Sciences, Yokohama National University, Yokohama, Japan \\ ${ }^{2}$ Faculty of Agriculture, Tokyo University of Agriculture and Technology, Fuchu, Tokyo, Japan \\ ${ }^{3}$ School of Agriculture, Utsunomiya University, Utsunomiya, Japan \\ Correspondence: Hamako Sasamoto, Faculty of Agriculture, Tokyo University of Agriculture and Technology, \\ Fuchu, Tokyo, 183-8509, Japan. Tel: 81-42-367-5674. E-mail: sasamoto@ynu.ac.jp
}

Received: June 15, 2021

doi:10.5539/jps.v10n2p8
Accepted: July 6, $2021 \quad$ Online Published: July 17, 2021

URL: https://doi.org/10.5539/jps.v10n2p8

\begin{abstract}
Allelopathic activities of a salt-tolerant and low-temperature tolerant mangrove tree, Kandelia obovata, which grows in brackish water regions of sub-tropical areas, and two fast-growing trees, Betula platyphylla and Populus alba, which grow in the temperate area, were examined by two in vitro bioassay methods, the sandwich method using dried leaves and the protoplast co-culture method using leaf protoplasts. Lettuce root growth examined by the sandwich method, was inhibited $50 \%$ by $50 \mathrm{mg}$ dried mature leaves of $K$. obovata. In the protoplast co-culture method, inhibition rates of cell division of lettuce protoplasts were $31 \%$ and $69 \%$ by leaf protoplasts of $K$. obovata at densities of $1 \times 10^{4} \mathrm{~mL}^{-1}$ and $5 \times 10^{4} \mathrm{~mL}^{-1}$, respectively. These results were compared with the inverse relationship between allelopathic activities and salt tolerance of mangrove plants of different families. B. platyphylla showed $37 \%$ inhibition by the sandwich method using dried young leaves, but only $10 \%$ inhibition at $5 \times 10^{4} \mathrm{~mL}^{-1}$ by the protoplast co-culture method using leaf protoplasts of $B$. platyphylla. Dried young leaves of $P$. alba showed $66 \%$ inhibition, but the leaf protoplasts at the density of $5 \times 10^{4} \mathrm{~mL}^{-1}$ showed highly stimulatory activity. Abscisic acid, of which contents in leaf protoplasts of three tree species varies from high to low in relation to salt tolerance and recalcitrance of tissue culture, was discussed as a putative allelochemical.
\end{abstract}

Keywords: abscisic acid, allelopathy, birch, leaf protoplast culture, poplar, salt-tolerant mangrove tree

\section{Introduction}

\subsection{In Vitro Bioassay Method of Allelopathy: the Sandwich Method}

Allelopathy is one of the strategies of plants, which cannot move, to survive by emitting allelochemicals to inhibit the growth of neighboring plants sharing the same habitat. In the broad definition of allelopathy, stimulatory effects among plants and microorganisms are also included (Fujii, 2000). As an in vitro bioassay method, the sandwich method (Fujii et al., 2003), which measures the effects of 10 and $50 \mathrm{mg}$ dried leaves, was used to test many plants including trees using lettuce as a recipient plant (Fujii, 2000; Bergum et al., 2019). Lettuce was the most sensitive among the recipient plant species examined including rice (Itani et al., 1998).

\subsection{In vitro Bioassay Method of Allelopathy: the Protoplast Co-culture Method}

The protoplast co-culture method was first developed to measure the effects of herbaceous leguminous plants (Mucuna pruriens) on the recipient lettuce or rice protoplasts. It has been applied to study the mechanism(s) of allelopathy at the cellular level and to simulate the possible future environmental risks in the field (Sasamoto $e t$ al., 2013). In the assay of herbaceous test plants, protoplasts were isolated from several tissues e.g., leaf (M. pruriens, Sasamoto et al., 2013; Arabidopsis thaliana, Sasamoto et al., 2017a,b); epicotyl and root (Vicia villosa, Sasamoto et al., 2019); cotyledons (Pueraria montana, invasive Kudzu, Kobayashi et al., 2021).

The number of plant species tested using the protoplast co-culture method is increasing, including woody and tree species, e.g., Mucuna gigantea, bamboo species, Prunus yedoensis, Coffea canephora, and an invasive leguminous tree, Leucaena leucocephala (Mori et al., 2015; Ogita and Sasamoto, 2017; Fujise et al., 2018; Ogita 
et al., 2020). Tissue cultured cells (calluses and suspension cultured cells) have been used for protoplast isolation of these tree test plants. Co-culture medium was $50 \mu \mathrm{L}$ liquid MS (Murashige and Skoog, 1962) basal medium containing $1 \mu \mathrm{M}$ 2,4-dichlorophenoxyacetic acid (2,4-D) and $0.1 \mu \mathrm{M}$ benzyladenine (BA), 3\% sucrose and different concentrations of osmoticum.

\subsection{Salt Tolerance of Mangrove Plants at the Cellular Level}

Mangrove plants, mainly tree species of different families, are distributed in brackish waters in tropical and subtropical areas (Tomlinson, 1986; Spalding et al., 2010). The highly salt-tolerant or halophilic nature of seaward-side grown species of Sonneratiaceae (Sonneratia alba) and Avicenniaceae (Avicennia alba) have been studied using tissue-cultured cells and their protoplasts (Kawana and Sasamoto, 2008; Hayashi et al., 2009; Hasegawa et al., 2013; Sasamoto et al., 2020). In addition to stimulation by $\mathrm{NaCl}$, different responses to other sea salt ions $\left(\mathrm{K}^{+}, \mathrm{Mg}^{2+}, \mathrm{Ca}^{2+}\right)$ added to MS basal medium were examined. Comparison of the responses of rice cells and lettuce protoplasts revealed specific $\mathrm{Ca}^{2+}$-related cellular mechanisms of halophilism (Hayatsu et al., 2014, 2017).

One of the Rhizophoraceae mangroves, Kandelia obovata (Sheue et al., 2003) previously known as $K$. candel in an in vitro culture study (Ogita et al., 2004), was transplanted to the temperate area in Japan (Masuda, 1999). It is a unique low temperature-tolerant mangrove (Kao et al., 2004; Suwa, 2014; Watanabe, personal communication). Studies on $K$. obovata leaf protoplasts revealed stimulation of growth by addition of high concentrations of $\mathrm{Mg}^{2+}$ ions, but inhibition by other cationic ions (Kaai et al., 2006).

\subsection{Allelopathy of Mangrove Plants}

An inverse relationship was found between salt tolerance and allelopathic activities among three Sonneratia mangrove tree species (Hasegawa et al., 2014), using two in vitro bioassay methods of allelopathy, the sandwich method using mature leaves, and the protoplast co-culture method. The trees are distributed from the seaward side (Sonneratia alba) to upstream side (S. caseolaris) and in between (S. ovata). High allelopathic activities were also found in the upstream-side, leguminous mangrove tree, Derris indica, in which an isoflavonoid, rotenone, was studied as a putative allelochemical (Inoue et al., 2015). Protoplasts of tissue cultured cells (suspension-cultured cells or calluses), not leaf protoplasts, of these mangroves were used in the protoplast co-culture method.

In the present study, the allelopathic activities were determined using mature leaves of the mangrove, Kandelia obovata by the sandwich method, and using leaf protoplasts of $K$. obovata by the protoplast co-culture method and compared with reports on other mangroves.

\subsection{Allelopathy of Fast-growing Trees, Betula platyphylla and Poplus alba}

In the present study, allelopathy of the young leaves of in vitro shoot cultures of two fast-growing broad-leaved trees, B. platyphylla (birch) and P. alba (poplar), which grow in the temperate regions, was examined by the sandwich method and compared with the allelopathy of leaf protoplasts examined by the protoplast co-culture method. These tree species had been well succeeded in regenerating plants from leaf protoplast cultures and in protoplast fusion studies of broad-leaved trees (Wakita et al., 2005, Sasamoto et al., 2006). Regenerated plants were obtained from very low numbers of electro-fused $P$. alba leaf protoplasts (Sasamoto et al., 2000).

The leaf protoplasts of $P$. alba showed a weak response to all added cationic ions, $\mathrm{Na}^{+}, \mathrm{K}^{+}, \mathrm{Mg}^{2+}, \mathrm{Ca}^{2+}$, in an $\mathrm{Cl}^{-}$ ion-depending manner under different medium pHs (Fukumoto et al., 2004). In leaf protoplast culture of $B$. platyphylla, tolerance to $\mathrm{Ca}^{2+}$ ion and acidic medium $\mathrm{pH}$ was reported (Seyama et al., 2008, though the protoplast culture methods were wrongly described).

\subsection{Evaluation of Abscisic Acid as a Putative Allelochemical}

Abscisic acid (ABA) is a stress-related growth retardant hormone. Exogenously supplied ABA inhibited the growth of leaf protoplasts of Populus alba (Sasamoto et al., 1995). The high ABA content of leaf protoplasts of Betula platyphylla was related to the recalcitrancy of their tissue culture (Sasamoto et al., 2002). On the contrary, the content of ABA in the cells of halophilic mangroves (Sonneratia alba, Avicennia marina, and A. alba) and effect of ABA on their cell growth in the culture medium varied from stimulatory to inhibitory (Kawana et al., 2009; Hasegawa et al., 2011). The leaf protoplasts of Kandelia obovata, showed a high ABA content and ABA added to the culture medium inhibited the growth of the protoplasts (Kaai et al., 2008).

In the protoplast co-culture bioassay of allelopathy, $\mathrm{ABA}$ in the culture medium stimulated the growth of recipient lettuce protoplasts, while the antagonistic plant hormone, gibberellic acid inhibited it (Sasamoto et al., 2013). 
In the present study, ABA is discussed as a putative allelochemical in leaf protoplasts of B. platyphylla, $P$. alba and $K$. obovata. The present results were compared with the reported strong allelopathic activity of protoplasts of suspension cultured cells of a non-mangrove tree, Prunus yedoensis (Fujise et al., 2018). In addition, the possibility of using of the protoplast co-culture method with digital image analysis (Sasamoto et al., 2017a) in the search for new allelochemicals in mangrove plant cells is discussed.

\section{Method}

\subsection{Materials}

Leaves of Kandelia obovata to be used in the sandwich method (2.2) were collected in Iriomote Island, Okinawa, Japan, washed, dried at $60^{\circ} \mathrm{C}$ for $18 \mathrm{hr}$ and stored in a dry condition until use. Leaves of $K$. obovata for protoplast isolation were obtained from potted plants grown as described previously (Kaai et al., 2008).

Young leaves of Betula platyphylla and Populus alba were obtained from shoot cultures, which were the same strains as used for protoplast cultures and cell fusion research (Sasamoto et al., 2000, 2002; Wakita et al., 2005; Sasamoto et al., 2006). Medium for shoot culture of P. alba, was MS (Murashige and Skoog, 1962) basal medium, containing $4 \mu \mathrm{M}$ indolebutyric acid (IBA), 3\% sucrose, $1 \%$ agar, at $\mathrm{pH} 5.9$ before autoclaving at $120^{\circ} \mathrm{C}$ for $20 \mathrm{~min}$. Medium for B. platyphylla was MS basal medium containing $2.5 \mu \mathrm{M}$ IBA, $0.1 \mu \mathrm{M}$ naphthalene acetic acid (NAA), $2 \%$ sucrose and $1 \%$ agar. Aseptic shoot cultures were incubated in the light condition (60 $\mu E$, 16-24 hr photoperiod) at $25^{\circ} \mathrm{C}$.

Aseptic seedlings of Lactuca sativa (lettuce) were prepared as described previously (Sasamoto et al., 2013). Briefly, lettuce seeds 'Great Lakes 366' were sterilized with $1.5 \% \mathrm{NaClO}$ solution for 15 min and washed with autoclaved water three times. They were cultured on $0.8 \%$ agar medium for one to three weeks in the light condition at $25^{\circ} \mathrm{C}$.

\subsection{Sandwich Method}

The sandwich method was performed as described previously (Fujii et al., 2003, 2004). Briefly, $10 \mathrm{mg}$ or $50 \mathrm{mg}$ of dried leaves were sandwiched between two layers of $5 \mathrm{~mL}$ of $0.5 \%$ agar (powder, gelling temp. $30-31^{\circ} \mathrm{C}$, Nacalai tesque Co. Ltd. Kyoto, Japan) in 6-well plates (Nunc ${ }^{\mathrm{TM}}$, ThermoFisher Scientific, Waltham, Masaachusetts, U.S.A.). Lengths of hypocotyls and roots of germinated seeds of lettuce on agar (five per one well) were measured after 3 days of incubation at $20^{\circ} \mathrm{C}$ in the dark. The control treatment consisted of seeds germinated in the absence of dried leaves. Data were recorded as percentage growth of the control and averaged with standard deviation.

\subsection{Protoplast Isolation and Purification}

All procedures were as described previously. Briefly, a $7 \mathrm{~cm}$-long leaf of $K$. obovata was sterilized with $1 \%$ $\mathrm{NaClO}$ solution for $40 \mathrm{~min}$, and protoplasts were isolated using 1\% each of Cellulase RS and Driselase 20 in 0.8 M mannitol solution at $30^{\circ} \mathrm{C}$ (Kaai et al., 2008). Protoplasts of B. platyphylla were isolated using $1 \%$ each of Cellulase R10 and Driselase 20 in $0.6 \mathrm{M}$ mannitol solution by overnight floating method (Wakita et al., 1996, 2005). Protoplasts of $P$. alba were isolated using $1 \%$ Cellulase RS, $0.25 \%$ Pectolyase Y-23 in $0.6 \mathrm{M}$ mannitol solution for $2 \mathrm{hrs}$ at static condition (Sasamoto et al., 2002, 2006). After passing through a 42-63 $\mu \mathrm{m}$ sized nylon mesh, the protoplasts were purified by washing three times with osmoticum solution followed by centrifugation at $100 \mathrm{~g}(800-900 \mathrm{rpm})$ for 3-5 min.

Protoplast isolation from cotyledons (and small leaf) of recipient lettuce was performed in a flask using $1 \%$ each of Cellulase RS and Macerozyme R-10 in the same osmoticum solution as of test plant protoplasts for 20-24 hrs (Sasamoto et al., 2013).

\subsection{Protoplast Co-culture with Lettuce Protoplasts}

Basically we used the method reported for herbaceous leguminous plants (Sasamoto et al., 2013). Test plant protoplasts with recipient lettuce protoplasts were co-cultured in liquid MS basal medium containing $1 \mu \mathrm{M}$ of 2,4-dichlorophenoxyacetic acid (2,4-D) and $0.1 \mu \mathrm{M}$ of benzyladenine (BA), 3\% sucrose and $0.6 \mathrm{M}$ mannitol solution.

Protoplast suspensions in osmoticum solution, $5 \mu \mathrm{L}$ each, were put into $50 \mu \mathrm{L}$ of liquid medium in a 96-well plastic culture plate (Falcon No. 3075); $100 \mu \mathrm{L}$ of autoclaved pure water (Milipore Direct-Q UV) was added to the space between the wells and the plate was tightly sealed with two layers of Parafilm ${ }^{\mathrm{R}}$. The protoplasts were cultured in the dark at $30^{\circ} \mathrm{C}(\mathrm{K}$. obovata, $)$, or at $28^{\circ} \mathrm{C}$ (B. platyphylla and $P$. alba) in a humid incubator $\left(\mathrm{CO}_{2}\right.$-incubator without the supply of $\mathrm{CO}_{2}$, APC-30DR, ASTEC Co. Ltd.). Numbers of non-spherically enlarged(E) and divided-protoplasts (D), and colonies composed of more than 4 cells (C) of lettuce were counted under 
an inverted microscope (Olympus CK40 or IX71) after 4 to 10 days of co-culture. The protoplast density of test plants ranged from $6-150 \times 10^{3} \mathrm{~mL}^{-1}$ (K. obovata), and 5-100 $\times 10^{3} \mathrm{~mL}^{-1}$ (B. platyphylla and $P$. alba). The recipient lettuce protoplast density ranged from $5-100 \times 10^{3} \mathrm{~mL}^{-1}$.

Lettuce protoplast growth was described as the $\%$ of control without test plant protoplasts. Data were averaged with standard errors at $5 \times 10^{3}-10^{5} \mathrm{~mL}^{-1}$ lettuce protoplast densities.

\section{Results and Discussion}

\subsection{Sandwich Method}
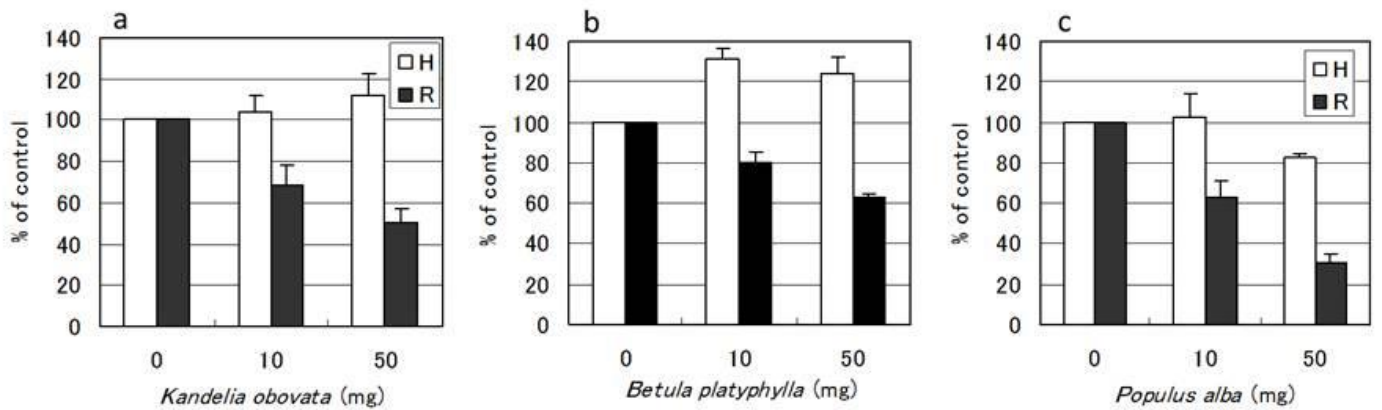

Figure 1. Effects of leaves of Kandelia obovata (a), Betula platyphylla (b) and Populus alba (c) on the growth of hypocotyls (white, $\mathrm{H}$ ) and of roots (black, $\mathrm{R}$ ) of lettuce seedlings measured by the sandwich method

As shown in Figure 1a, $50 \mathrm{mg}$ of mature leaves of the mangrove, Kandelia obovata inhibited the root elongation of lettuce seedlings $50 \%$. This was similar to the $53 \%$ inhibition obtained with potted plants of $K$. obovata (Hayashi et al., 2008). This value was similar to that obtained for the group 2 mangroves, and higher than that obtained for group 1 (the seaward-side grown, highly salt tolerant, low allelopathic activity, less than $45 \%$ inhibition), but lower than that obtained for group 3 (the upstream grown, less salt tolerant, highly allelopathic, more than 80\% inhibition) (Sasamoto and Hasegawa, 2014, Sasamoto et al., 2014). Group 1 included Sonneratia alba (Hasegawa et al., 2014), and group 3 included Derris indica (Inoue et al., 2015) and S. caseolaris (Hasegawa et al., 2014). In the group 3 mangrove species, the growth of both hypocotyls and roots of lettuce seedling were strongly inhibited. In contrast, K. obovata (Figure 1a) did not inhibit the growth of the hypocotyls of lettuce.

A non-mangrove, invader tree, Leucaena leucocephala, which grows in sub-tropical areas, strongly inhibited the growth of lettuce root and hypocotyl $(88 \%$ and $91 \%$ by $50 \mathrm{mg}$ ) examined by the sandwich method. Lettuce root growth was inhibited $76 \%$ by even $10 \mathrm{mg}$ (Mori et al., 2015). Such inhibition was similar or stronger than that of the group 3 mangrove species.

Two fast-growing non-mangrove, tree species, Betula platyphylla and Populus alba, grow in temperate regions. As shown in Figure 1b, $50 \mathrm{mg}$ of young leaves of B. platyphylla moderately inhibited lettuce root growth (37\%), but stimulated the growth of hypocotyls. Mature leaves of $B$. platyphylla were reported to inhibit the growth of lettuce roots only $3 \%$ (Fujii, 2000). These trees inhibited growth less than the group 1 mangrove trees. As shown in Figure 1c, $50 \mathrm{mg}$ of young leaves of Populus alba inhibited the growth of lettuce roots (69\%), but had no effect on hypocotyls. Such moderate inhibition (56\%) was reported for mature leaves of another poplar species, Populus nigra (Fujii, 2000). These values are similar to those obtained for the group 2 mangroves.

\subsection{Protoplast Co-culture Method}

\subsubsection{Leaf Protoplasts of Kandelia obovata}

Figure 2 shows the results of the protoplast co-culture of the leaf protoplasts of the mangrove species, $K$. obovata. After 10 days of co-culture, colony formation of lettuce protoplasts was inhibited depending on leaf protoplast densities of $K$. obovata. Calculated values of inhibition at $1 \times 10^{4} \mathrm{~mL}^{-1}$ and at $5 \times 10^{4} \mathrm{~mL}^{-1}$ of $K$. obovata were $31 \%$ and $69 \%$, respectively.

These inhibition rates were in between those of the group 1 mangrove, Sonneratia alba $(24 \%, 75 \%)$, and the group 3 mangroves, S. caseolaris $(50 \%, 92 \%)$ and D. indica $(58 \%, 99 \%)$ of previous reports (Hasegawa et al., 2014; Sasamoto et al., 2014; Inoue et al., 2015). These were obtained using protoplasts of suspension cultured cells or callus. 


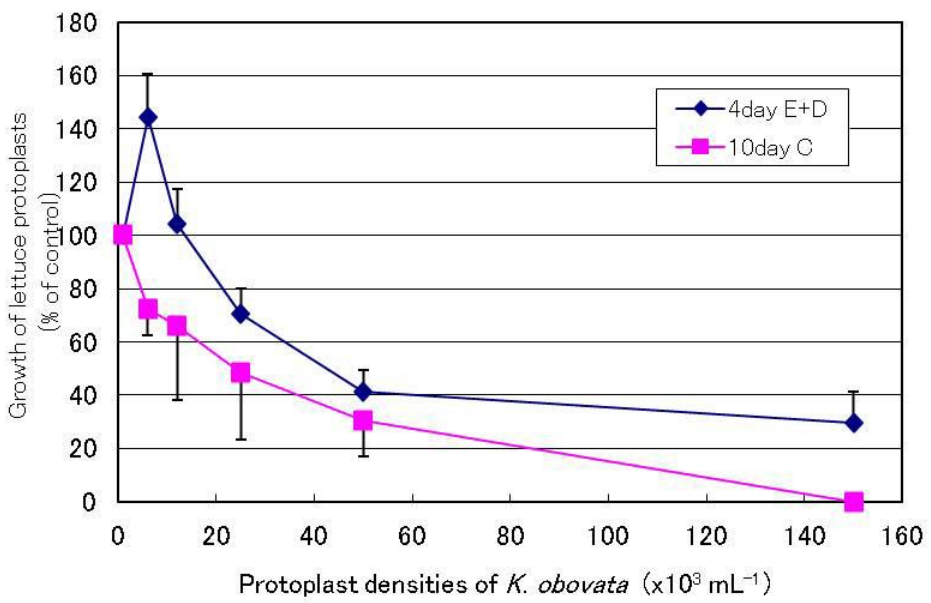

Figure 2. Activity of leaf protoplasts of mangrove species, Kandelia obovata, on lettuce protoplast growth at early cell division stage (day 4, diamond) and colony formation stage (day 10, square) measured by the protoplast co-culture method. The medium was MS basal medium containing $1 \mu \mathrm{M}$ 2,4-D, $0.1 \mu \mathrm{M}$ BA, 3\% sucrose, and $0.64 \mathrm{M}$ mannitol

The protoplast co-culture method has been improved by averaging the \% of growth of control without test plants at different lettuce protoplast densities $\left(5-100 \times 10^{3} \mathrm{~mL}^{-1}\right)$ as described in 2.4. (Sasamoto and Ashihara, 2014; Sasamoto et al., 2015). There was a tendency of variation in the inhibition $\%$ with the lettuce protoplast density.

After 4 days of co-culture, at the early cell division stage of lettuce, including the numbers of non-spherically enlarged lettuce protoplasts (E, cell wall formed), $44 \%$ stimulation of lettuce growth was observed at $6 \times 10^{3}$

$\mathrm{mL}^{-1}$ of $K$. obovata (Figure 2). Such patterns of stimulation at low protoplast densities and inhibition at high protoplast densities of test plant are similar to protoplasts of suspension cultured cells of three bamboo species (Ogita and Sasamoto, 2017) and protoplasts of calluses of coffee (Ogita et al., 2020). Inhibition values of the non-mangrove invader tree, Leucaena leucocephala were $99 \%$ and $100 \%$ at $1 \times 10^{4} \mathrm{~mL}^{-1}$ and at $5 \times 10^{4} \mathrm{~mL}^{-1}$, respectively, after 4 days of co-culture (Mori et al., 2015).

\subsubsection{Leaf Protoplasts of Betula platyphylla}

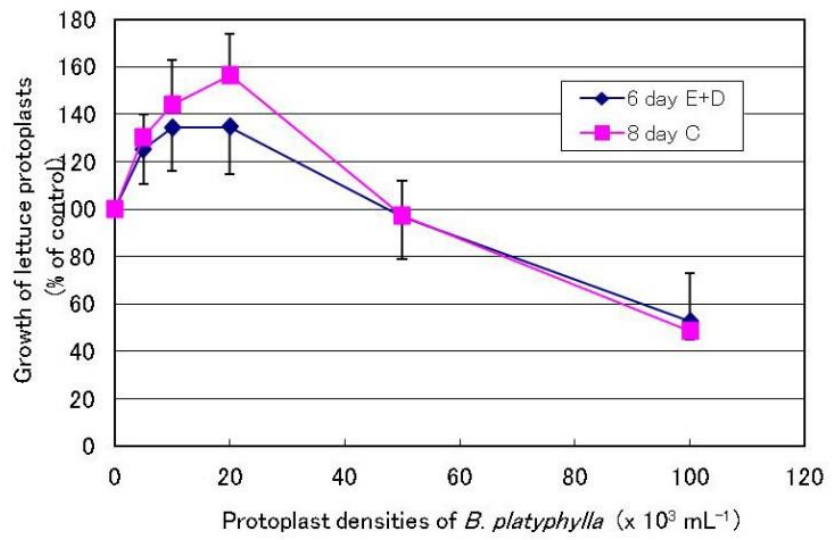

Figure 3. Activity of leaf protoplasts of fast-growing tree, Betula platyphylla on lettuce protoplast growth at early cell division stage (day 6 , diamond) and colony formation stage (day 8 , square) measured by the protoplast co-culture method. The medium was MS basal medium containing $1 \mu \mathrm{M}$ 2,4-D, $0.1 \mu \mathrm{M} \mathrm{BA}$, $3 \%$ sucrose, and $0.6 \mathrm{M}$ mannitol

The allelopathy of the non-mangrove tree species, B. platyphylla was examined using the protoplast co-culture 
method. As shown in Figure 3, after 6 days of co-culture, at the early cell division stage of lettuce protoplasts, $35 \%$ stimulation was observed at $1 \times 10^{4} \mathrm{~mL}^{-1}$, and $47 \%$ inhibition at $1 \times 10^{5} \mathrm{~mL}^{-1}$. Such pattern of stimulation at low protoplast densities and inhibition at high protoplast densities of test plant protoplasts were also observed in the mangrove, $K$. obovata (Figure 2).

At the colony formation stage of lettuce ( 8 days of co-culture), colony formation was stimulated $51 \%$ at the density of $1 \times 10^{4} \mathrm{~mL}^{-1}$, and was inhibited $54 \%$ at the density of $1 \times 10^{5} \mathrm{~mL}^{-1}$. This is different from the results obtained with $K$. obovata, which showed only inhibition.

\subsubsection{Leaf Protoplasts of Populus alba}

Protoplasts of non-mangrove tree species, $P$. alba were co-cultured. As shown in Figure 4, strong stimulation (100-200\%) of lettuce protoplast growth was observed at the colony formation stage of lettuce. Similar but less stimulation (up to $46 \%$ ) was also observed at the early cell division stage of lettuce after 6 days of co-culture. Similar $40 \%$ stimulation at the early cell wall formation stage of lettuce, at up to $1 \times 10^{5} \mathrm{~mL}^{-1}$, was reported for the protoplasts of yellow callus of a halophilic mangrove Avicennia alba. However, inhibition was reported at the cell division stage at all protoplast densities tested (Sasamoto et al., 2020). Recently, protoplasts of embryogenic coffee callus cells were reported to show $150 \%$ stimulation at the early cell wall formation stage, and moderate inhibition at the cell division stage (Ogita et al., 2020). Compared with the responses of the protoplasts of these two trees, the leaf protoplasts of $P$. alba showed very strong stimulation at the colony formation stage.

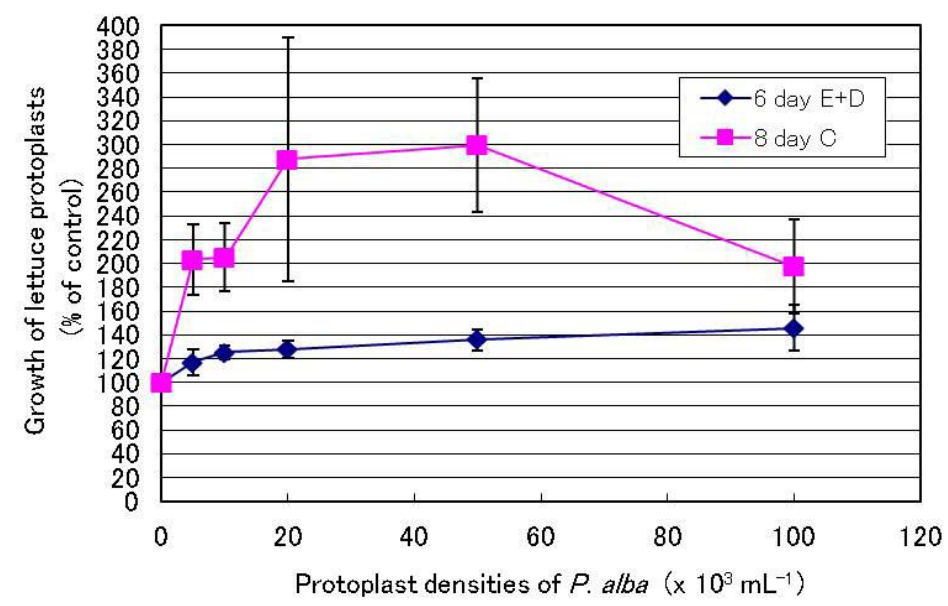

Figure 4. Activity of leaf protoplasts of fast-growing tree, Populus alba, on lettuce protoplast growth at early cell division stage (day 6, diamond) and colony formation stage (day 8 , square) measured by the protoplast co-culture method. The medium was MS basal medium containing $1 \mu \mathrm{M}$ 2,4-D, $0.1 \mu \mathrm{M} \mathrm{BA}, 3 \%$ sucrose, and $0.6 \mathrm{M}$ mannitol

\subsection{Sandwich Method and Protoplast Co-culture Method}

\subsubsection{Kandelia obovata}

Both mature leaves (Figure 1a) and leaf protoplasts (Figure 2) of the mangrove, $K$. obovata showed moderate inhibitory allelopathic activities by the sandwich method and protoplast co-culture method, respectively, similar to the group 2 mangroves, between group 1 (the seaward-side grown, highly salt tolerant), and group 3 (the upstream grown, less salt tolerant) (Sasamoto et al., 2014).

\subsubsection{Fast Growing Trees or Woody Species}

Betula platyphylla showed moderate inhibition on lettuce root growth by the sandwich method (Figure 1b). However, inhibition by leaf protoplast of B. platyphylla was observed only at a high protoplast density, $1 \times 10^{5}$ $\mathrm{mL}^{-1}$, by the protoplast co-culture method; and stimulation was observed at low protoplast densities (Figure 3 ).

Although Populus alba showed moderate inhibition of lettuce root growth by the sandwich method (Figure 1c), strong stimulation was observed by the protoplast co-culture method (Figure 4).

Different inhibition patterns between protoplast co-culture method and the sandwich method were also seen in four bamboo species (Bambusa multiplex, Phyllostachys bambusoides, Phyllostachys nigra, Sasa kurilensis; Ogita and Sasamoto 2017). All four bamboo species showed moderate inhibition by the sandwich method. 
However, Sasa kurilensis showed strong inhibition by the protoplast co-culture method, and the other three bamboo species showed stimulation at lower protoplast densities. Such a discrepancy might be explained by the differences of endogenous levels of putative allelochemical(s) among protoplasts of young and mature leaves of test plants. Another explanation is the use of dead leaf leachates for the sandwich method and living cells for the protoplast co-culture method (Ogita and Sasamoto, 2017). In Sasa species, another bioassay method of allelopathy, the plant box method, which measures the effect of exudates from intact roots (Fujii, 2000, Fujii et al., 2007), was similarly inhibitory as in the protoplast co-culture method. Though, small seedlings are needed for the plant box method, and it is difficult to obtain them in tree species all year round.

\subsection{Abscisic Acid (ABA) as a Putative Allelochemical in Protoplasts}

\subsubsection{ABA in Betula platyphylla and Populus alba}

ABA is a plant hormone related to stress tolerance and known as a growth retardant. Exogenously supplied ABA inhibited the growth of leaf protoplasts of $P$. alba (Sasamoto et al., 1995). Protoplast culture of B. platyphylla was recalcitrant to antagonize endogenous $\mathrm{ABA}$, when the strong cytokinins, thidiazuron or $N$-(2-chloro-4-pyridyl)- $N$ '-phenylurea (4-PU or CPPU) were not used (Wakita et al., 1996). Very high content of ABA in leaf protoplasts of $B$. platyphylla ( 16.7 pmoles / $6 \times 10^{6}$ protoplasts $)$ and low content $(0.81$ pmoles / $6 \times$ $10^{6}$ protoplasts) in leaf protoplasts of $P$. alba were found using micro scale extraction, partition, and ELISA test (Sasamoto et al., 2002). As the diameter of these protoplasts was $20 \mu \mathrm{m}$, the calculated ABA concentration in each protoplast of Populus alba was $0.03 \mu \mathrm{M}$, and $0.67 \mu \mathrm{M}$ in B. platyphylla protoplast (Table 1).

Table 1. Calculated concentrations of ABA in leaf protoplasts

\begin{tabular}{lccl}
\hline Plant species & $\mathrm{ABA}(\mu \mathrm{M})$ & $\mathrm{ABA}\left(\mathrm{pmoles} / 6 \times 10^{6}\right.$ protoplasts) & References \\
\hline Populus alba & 0.03 & 0.81 & Sasamoto et al. 2002 \\
Betula platyphylla & 0.67 & 16.7 & Sasamoto et al. 2002 \\
Kandelia obovata & 0.36 & 8.92 & Kaai et al. 2008 \\
\hline
\end{tabular}

*Diameter of protoplasts was $20 \mu \mathrm{m}$.

However, co-cultured poplar leaf protoplasts stimulated the growth of lettuce (Figure 4). The growth of recipient lettuce protoplasts was stimulated by exogenously supplied ABA at $0.1 \mu \mathrm{M}$ up to $10 \mu \mathrm{M}$; the highest stimulation at $1 \mu \mathrm{M}$ (Sasamoto et al., 2013). Growth of lettuce protoplasts was strongly stimulated by co-culture with $P$. alba leaf protoplasts (Figure 4). Co-culture with B. platyphylla was stimulatory at low densities and inhibitory at a high density (Figure 3). This might be explained by the low and high ABA content of protoplasts of these two trees. Therefore, ABA in leaf protoplasts of $P$. alba and B. platyphylla might act as an allelochemical on lettuce protoplast growth in the protoplast co-culture method.

On the other hand, ABA has been reported to have a moderate inhibitory effect on seedling growth of recipient lettuce (Hiradate et al., 2005). However, the effect of ABA alone could not explain the stronger inhibition of $P$. alba (Figure 1c) than that of B. platyphylla (Figure 1b) on the lettuce root growth examined by the sandwich method. Such discrepancy might be partly explained by the differences in endogenous levels of ABA and antagonistic plant hormones among protoplasts and seedlings of lettuce. In P. alba, exogenous ABA inhibited the growth of leaf protoplasts. However, exogenous ABA stimulated the protoplast growth of suspension cultured cells of $P$. alba, to antagonize the high endogenous content of gibberellins (Sasamoto et al., 1995).

Very strong inhibitory allelopathic activity was reported in a flower tree, Prunus yedoensis using both the protoplast co-culture method and the sandwich method (Fujise et al., 2018). Though exogenous $1 \mu \mathrm{M} \mathrm{ABA}$ strongly inhibited the growth of Prunus protoplasts themselves, low calculated content of ABA $(0.09 \mu \mathrm{M})$ in isolated protoplasts of suspension cells of Prunus yedoensis (Yokota et al., 2005) was not sufficient to be considered as a cause of strong inhibitory allelopathic activity of Prunus protoplasts on lettuce protoplasts, and coumarin was suggested as an allelochemical in Prunus protoplasts.

\subsubsection{ABA in Kandelia obovata}

The inhibitory effect of $\mathrm{ABA}$ in tissue culture has also been reported in the mangrove, $K$. obovata leaf protoplasts, along with high endogenous ABA content in leaf protoplasts (8.92 pmoles / $6 \times 10^{6}$ protoplasts) (Kaai et al., 2008). As the diameter of $K$. obovata leaf protoplast was $20 \mu \mathrm{m}$, the calculated ABA concentration in each protoplast of $K$. obovata was $0.36 \mu \mathrm{M}$ (Table 1). This value was several times higher than that of $P$. alba leaf protoplasts (Sasamoto et al., 1995), but several times lower than that of B. platyphylla leaf protoplasts (Sasamoto et al., 2002). 
The leaf protoplasts of $K$. obovata showed inhibitory allelopathic activities at $1 / 10$ density of B. platyphylla, by the protoplast co-culture method (Figure 2). Accordingly, the high ABA content calculated in isolated protoplasts of $K$. obovata, could not be the single and direct cause of the moderate inhibitory allelopathic activity. Different allelochemical(s), which mimic the stimulatory effect of ABA, might exist in $K$. obovata protoplasts.

\subsection{Finding Allelochemicals in Mangrove Plant Cells Using Protoplast Co-culture Method}

The contents of ABA in the protoplasts of halophilic mangroves (Sonneratia alba, Avicennia alba, A. marina) varied from high to low, and the growth of protoplasts has been reported to be inhibited or stimulated by ABA in the culture medium (Kawana et al., 2009; Hasegawa et al., 2011). Though, an inverse relationship was found between allelopathic activity and salt tolerance of Sonneratia mangrove plants (Hasegawa et al., 2014), inhibitory allelopathic activities were found in all mangroves tested using both the sandwich method and the protoplast co-culture method (Figure 1a, Figure 2; Mori et al., 2012; Sasamoto et al., 2014; Inoue et al., 2015). Some allelochemical(s) with inhibitory activities, which mimic the stimulatory activity of ABA, might be found even in the seaward-side grown mangrove species using the protoplast co-culture method.

The content of a putative allelochemical, ABA, in protoplasts was analyzed using the small-scale extraction and fractionation (purification) method in combination with the sensitive assay method (ELISA test; Sasamoto et al., 2002). In the protoplast co-culture method for the assay of allelopathic activities, the amount of chemical(s) in a $50 \mu \mathrm{L}$ medium, and the protoplast numbers needed are very low (500 protoplasts at $1 \times 10^{4} \mathrm{~mL}^{-1}$, and 2500 at 5 $\left.\times 10^{4} \mathrm{~mL}^{-1}\right)$. In addition to the chemicals, already found in tissues of test plants, or in tissue cultured cells, finding of new allelochemicals might be possible by using a combination of such small-scale methods. A single chemical of large amount might not be the only cause of strong inhibitory allelopathic activity of plants (Kobayashi et al., 2021).

Recently, digital image analysis has been applied to the protoplast co-culture method (DIA-PP method) for leaf protoplasts of a herbaceous plant, Arabidopsis thaliana using lettuce as a recipient (Sasamoto et al., 2017a,b). In addition to the effects on cell wall formation and cell division stages of protoplasts of the recipient plants, effects on the yellow pigment accumulation stage, which is specific to recipient lettuce protoplasts after 3 weeks to 2 months of co-culture, was quantitatively analyzed by digital image analysis. Using the DIA-PP method, a carotenoid, neoxanthin, was found as an allelochemical of yellow callus of a group 1 mangrove, Avicennia alba. Strong inhibition at the cell division stage, and moderate inhibition at the yellow pigment (including a carotenoid) accumulation stage were observed (Sasamoto et al., 2020; Sasamoto et al., 2021). The yellow callus was not yellow in the original cultured cells after induction in the dark (Hasegawa, 2014; Tsuchiya et al., 2013), of which protoplasts were highly salt tolerant (Hasegawa et al., 2013). In addition, using the DIA-PP method, an anthocyanin, cyanidin 3,5-di- $O$-glucoside, was found as an allelochemical of red callus of a less salt-tolerant, group 2 mangrove, S. ovata (Hasegawa et al., 2014; Sasamoto et al., 2014; Sasamoto et al., 2018). Moderate inhibition of cell division and no inhibition at the yellow pigment accumulation stage were observed in co-cultured protoplasts of S. ovata (Sasamoto et al., 2018). Similarly, no inhibition at the yellow pigment accumulation stage was observed in the DIA-PP method of Kandelia obovata (Sasamoto et al., in preparation).

Protoplast co-culture method and DIA-PP method will contribute to the understanding of the underlying mechanism(s) of allelopathy at a cellular level, and they can be applied under different culture conditions, e.g., high and low temperatures, and different salts conditions, in order to simulate the possible environmental risks of genetically modified plants and invasive plants (Fujise et al., 2012; Sasamoto et al., 2013; Suzuki et al., 2018).

\section{References}

Bergum, K., Shammi, M., Hasan, N., Asaduzzaman, M., Appiah, K. S., \& Fujii, Y. (2019). Potential allelopathic candidates for land use and possible sustainable weed management in south Asian ecosystem. Sustainability, 11, 2649. https://doi.org/10.3390/su11092649

Fujii, Y. (2000). Allelopathy, Nobunkyo, Tokyo. In Japanese.

Fujii, Y, Parvez, S. S., Parvez, M. M., Ohmae, Y., \& Iida, O. (2003). Screening of 239 medicinal plant species for allelopathic activity using the sandwich method. Weed Biol. and Manag, 3, 233-241. https://doi.org/10.1046/j.1444-6162.2003.00111.x

Fujii, Y., Shibuya, T., Nakatani, K., Itani, T., Hiradate, S., \& Parvez, M.M. (2004). Assesment method for allelopathic effect from leaf litter leachates. Weed Biol. Manag, 4, 19-23. https://doi.org/10.1111/j.1445-6664.2003.00113.x

Fujii, Y., Pariasca, D., Shibuya, T., Yasuda, T., Kahn, B., \& Waller, G.R. (2007). Plant Box Method: A Specific Bioassay to Evaluate Allelopathy through Root Exudates. In Y. Fujii \& S. Hiradate (Eds.), Allelopathy (pp. 
39-56). New Concepts and Methodology, Science Publishers, Inc., Enfield.

Fujise, K., Fukumoto, T., \& Sasamoto, H. (2012). Development of bioassay method of allelopathy in Prunus species using the protoplast co-culture method. Proceedings of the 76th Annual Meeting of the Botanical Society of Japan, Himeji.

Fujise, K., Yokota, S., \& Sasamoto, H. (2018). Evaluation of allelochemicals, abscisic acid and coumarin, in leaf-origin suspension cultured cells of Prunus yedoensis using protoplast co-culture bioassay method. Am. J. Plant Sci., 9, 172-184. https://doi.org/10.4236/ajps.2018.92015

Fukumoto, T., Nakamura, T., Suzuki, M., Ogita, S., Mimura, T., \& Sasamoto, H. (2004). Different effects of four salts and pHs on protoplast cultures of a mangrove, Bruguiera sexangula suspension cells, Populus alba leaves and tobacco BY-2 cells. Plant Biotechnol., 21(3), 177-182. https://doi.org/10.5511/plantbiotechnology.21.177

Hasegawa, A. (2014). Research on the relationship between salts tolerance and allelopathy at the cellular level in mangrove plants. Ph.D. thesis of Yokohama National University. p. 1-124.

Hasegawa, A., Hayashi, S., Kurita, A., Kaai, F., Kawana, Y., Fukumoto, T., \& Sasamoto, H. (2011). Stimulatory and inhibitory effects of abscisic acid on cell growth in protoplast cultures and the relation to its endogenous levels in Avicenniaceae mangrove cells. Mangrove Science, 8, 11-18.

Hasegawa, A., Kurita, A., Hayashi, S., Fukumoto, T., \& Sasamoto, H. (2013). Halophilic and salts tolerant protoplast cultures of mangrove plants, Sonneratia alba and Avicennia alba. Plant Biotechnol. Rep., 7, 205-209. https://doi.org/10.1007.s11816-012-0251-2

Hasegawa, A., Oyanagi, T., Minagawa, R., Fujii, Y., \& Sasamoto, H. (2014). An inverse relationship between allelopathic activity and salt tolerance in suspension cultures of three mangrove species, Sonneratia alba, $S$. caseolaris and $S$. ovata: Development of a bioassay method for allelopathy, the protoplast co-culture method. J. Plant Res., 127, 755-761. https://doi.org/10.1007/s10265-014-0651-1

Hayashi, S., Hasegawa, A., Yamamoto, R., Minagawa, R., Fujii, Y., \& Sasamoto, H. (2008). In vitro bioassay of allelopathy of mangrove plant species using the sandwich method. Abstract of the 14th Annual Meeting of Japan Society for Mangroves. p. 13.

Hayashi, S., Kuriyama, S., Kawana, Y., Hasegawa, A., Kurita, A., Minagawa, R., \& Sasamoto, H. (2009). Stimulatory effects of sea salts on cell growth in liquid culture of Avicenniaceae mangrove. Plant Biotechnol., 26, 561-564. https://doi.org.10.5511.plantbiotechnology.26.561

Hayatsu, M., Suzuki, S., Hasegawa, A., Tsuchiya, S., \& Sasamoto, H. (2014). Effect of $\mathrm{NaCl}$ on ionic content and distribution in suspension-cultured cells of the halophyte Sonneratia alba versus the glycophyte Oryza sativa. J. Plant Physiol., 171, 1385-1391. https://doi.org/10.1016/j.jplph.2014.06.008

Hayatsu, M., Suzuki, S., Tsuchiya, S., \& Sasamoto, H. (2017). Cellular calcium distribution modulates the growth of callus and protoplasts of halophyte mangrove plant, Avicennia alba-an X-ray microanalysis. $J$. Plant Stud., 6, 18-27. https://doi.org/10.5539/jps.v6n2p18

Hiradate, S., Morita, S., Furubayashi, A., Fujii, Y., \& Harada, J. (2005). Plant growth inhibition by cis-cinnamoyl glucosides and cis-cinnamic acid. J. Chem. Ecol., 31, 591-601. https://doi.org/10.1007/s10886-005-2047-0

Inoue, A., Mori, D., Minagawa, R., Fujii, Y., \& Sasamoto, H. (2015). Allelopathy in a leguminous mangrove plant, Derris indica: Protoplast co-culture bioassay and rotenone effect. Nat. Prod. Commun., 10(5), 747-750. https://doi.org/10.1177/1934578X1501000512

Itani, T., Hirai, K., Fujii, Y., Kohdai, H., \& Tamaki, M. (1998). Screening for allelopathic activity among weeds and medicinal plants using the "Sandwich Method". J. Weed Sci. Tech., 43, 258-266. https://doi.org/10.3719/weed.43.258

Kaai, F., Kawana, Y., \& Sasamoto, H. (2006). Endogenous levels of abscisic acid and gibberellins and recalcitrancy of leaf culture of a mangrove tree, Kandelia obovata. Abstract of 11th IAPTC\&B congress P1361. p. 138.

Kaai, F., Kawana., Y., \& Sasamoto, H. (2008). The relation between recalcitrancy of a mangrove plant, Kandelia obobata, and high endogenous level of abscisic acid. Plant Cell Tiss. Org. Cult., 94, 125-130. https://doi.org/10.1007/s11240-008-9394-9

Kao, W-U., Shih, C-N., \& Tsai, T-T. (2004). Sensitivity to chilling temperatures and distribution differ in the 
mangrove species Kandelia candel and Avicennia marina. Tree Physiol., 24, 859-864. https://doi.org/10.1093/treephys/24.7.859

Kawana, Y., \& Sasamoto, H. (2008). Stimulation effects of salts on growth in suspension culture of a mangrove plant, Sonneratia alba, compared with another mangrove, Bruguiera sexangula and non-mangrove tobacco BY-2 cells. Plant Biotechnol., 25, 151-155. https://doi.org.10.5511.plantbiotechnology.25.151

Kawana, H., Kaai, F., \& Sasamoto, H. (2009). Abscisic acid stimulates cell divisions in cultures of protoplasts isolated from cotyledons and suspension cells of a mangrove plant, Sonneratia alba: Small-scale measurements of abscisic acid and gibberellins in protoplasts. Mangrove Science, 6, 9-15.

Kobayashi, K., Sasamoto, H., Sasamoto, Y., Sugiyama, A., \& Fujii, Y. (2021). Evaluation of isoflavones as allelochemicals with strong allelopathic activities of Kudzu using protoplast co-culture method with digital image analysis. Am. J. Plant Sci., 12, 376-393. https://doi.org/10.4236/ajps.2021.123024

Masuda, S. (1999). Plantation of mangrove at the Aono river in MinamiIzu, Shizuoka Prefecture, Japan. MACRO REVIEW, 11, 63-70. https://doi.org/10.11286/jmr1988.11.2_63

Mori, D., Hasegawa, A., Fukumoto, T., Watanabe, S., \& Sasamoto, H. (2012). Protoplast co-culture bioassay for allelopathy in Bruguiera sexangula and Kandelia obovata. Abstract of the 18th Annual Meeting of Japan Society for Mangroves. p. 17.

Mori, D., Ogita, S., Fujise, K., Inoue, A., \& Sasamoto, H. (2015). Protoplast co-culture bioassay for allelopathy in leguminous plants, Leucaena leucocephala and Mucuna gigantea, containing allelochemical amino acids, mimosine and L-DOPA. J. Plant Stud., 4, 1-11. https://doi.org/10.5539/jps.v4n1p1

Murashige, T., \& Skoog, F. (1962). A revised medium for rapid growth and bio assays with tobacco tissue cultures. Physiol. Plant, 15, 473-497. https://doi.org/10.1111/j.1399-3054.1962.tb08052.x

Ogita, S., Yeung, E. C., \& Sasamoto, S. (2004). Histological analysis in shoot organogenesis from hypocotyls explants of Kandelia candel (L.) Druce. J. Plant Res., 117, 457-464. https://doi.org/10.1007/s10265-004-0180-4

Ogita, S., \& Sasamoto, H. (2017). In vitro bioassay of allelopathy in four bamboo species; Bambusa multiplex, Phyllostachys bambusoides, P. nigra, Sasa kurilensis, using sandwich method and protoplast co-culture method with digital image analysis. Am. J. Plant Sci., 8, 1699-1710. https://doi.org/10.4236/ajps.2017.87117

Ogita, S., Asrori, M. I., \& Sasamoto, H. (2020). Establishment of pluripotent cell cultures to explore allelopathic activity of coffee cells by protoplast co-culture bioassay method. Plants, 9, 1170. https://doi.org/10.3390/plants9091170

Sasamoto, A., \& Ashihara, H. (2014). Effect of nicotinic acid, nicotinamide and trigonelline on the proliferation of lettuce cells derived from protoplasts. Phytochem. Lett., 7, 38-41. https://doi.org/10.1016/j.phytol.2013.09.008

Sasamoto, H., \& Hasegawa, A. (2014). In vitro bioassay of allelopathy in mangrove plants. Abstract of the 20th Annual Meeting of Japan Society for Mangroves. p. 9.

Sasamoto, H., Hosoi, Y., \& Koshioka, M. (1995). Endogenous levels of four plant hormones may affect the culture conditions of poplar protoplasts to regenerate plants. Current Issues in Plant Molecular and Cellular Biology, Kluwer Academic Publishers. p. 481-486. https://doi.org/10.1007/978-94-011-0307-7_66

Sasamoto, H., Wakita, Y., Yokota, S., \& Yoshizawa, N. (2000). Large electro-fused protoplasts of Populus alba selected by a micromanipulator: Techniques and some characteristics of cells and their regenerants. J. For. Res., 5(4), 265-270. https://doi.org/10.1007/BF02767120

Sasamoto, H., Ogita, S., Wakita, Y., \& Fukui, M. (2002). Endogenous levels of abscisic acid and gibberellins in leaf protoplasts competent for plant regeneration in Betula platyphylla and Populus alba. Plant Growth Regul., 38, 195-201. https://doi.org/10.1023/A:1021515630671

Sasamoto, H., Wakita, Y., Yokota, S., Yoshizawa, N., Katsuki, T., Nishiyama, Y., Yokoyama, T., \& Fukui, M. (2006). Effects of electric cell fusion treatment among leaf protoplasts of Populus alba and Alnus firma on growth, leaf morphology, and RAPD pattern of eleven acclimatized plants. In Vitro Cell. Dev. Biol. Plant, 42, 174-178. https://doi.org/10.1079/IVP2005732

Sasamoto, H., Murashige-Baba, T., Inoue, A., Sato, T., Hayashi, S., \& Hasegawa, A. (2013). Development of a new method for bioassay of allelopathy using protoplasts of a leguminous plant Mucuna pruriens with a 
high content of the allelochemical L-DOPA. J. Plant Stud., 2, 71-80. https://doi.org/10.5539/jps.v2n2p71

Sasamoto, H., Hasegawa, A., Mochida, Y., Yokota, S., Minagawa, R., \& Fujii, Y. (2014). In vitro bioassay of allelopathy in mangrove species and Populus and Betula species: Sandwich method and protoplast co-culture method. Proceeding of the 78th Annual Meeting of the Botanical Society of Japan. p. 238.

Sasamoto, H., Fujii, Y., \& Ashihara, H. (2015). Effect of purine alkaloids on the proliferation of lettuce cells derived from protoplasts. Nat. Prod. Commun., 10, 751-754. https://doi.org/10.1177/1934578X1501000513

Sasamoto, H., Azumi, Y., \& Suzuki, S. (2017a). Development of a High-throughput Bioassay method of Allelopathy - Protoplast Co-culture and Digital Image Analysis-. Science Journal of Kanagawa University, 28, 63-70.

Sasamoto, H., Azumi, Y., Shimizu, M., Hachinohe, Y., \& Suzuki, S. (2017b). In vitro bioassay of allelopathy of Arabidopsis thaliana by sandwich method and protoplast co-culture method with digital image analysis. Plant Biotechnol., 34, 199-202. https://doi.org/10.5511/plantbiotechnology.17.1204a

Sasamoto, H., Iwashina, T., Suzuki, S., Azumi, Y., \& Fujii, Y. (2018). Evaluation of an anthocyanin, cyanidin 3,5-di-O-glucoside, as an allelochemical in red callus of a mangrove Sonneratia ovata, using protoplast co-culture bioassay method with digital image analysis. J. Plant Stud., 7, 1-10.

https://doi.org/10.5539/jps.v7n2p1

Sasamoto, H., Mardani, H., Sasamoto, Y., Wasano, N., Murashige-Baba, T., Sato, T., Hasegawa, A., \& Fujii, Y. (2019). Evaluation of canavanine as an allelochemical in etiolated seedlings of Vicia villosa Roth: protoplast co-culture method with digital image analysis. In Vitro Cell. Dev. Biol. Plant, 55, 296-304. https://doi.org/10.1007/s11627-019-09985-3

Sasamoto, H., Hayatsu, M., \& Suzuki, S. (2020). High allelopathic activity of carotenoid-accumulating callus of a halophilic mangrove plant, Avicennia alba: Protoplast co-culture method with digital image analysis. $J$. Plant Stud., 9, 1-12. https://doi.org/10.5539/jps.v9n1p1

Sasamoto, H., Suzuki, S., Mardani-Korrani, H., Sasamoto, Y., \& Fujii, Y. (2021). Allelopathic activities of three carotenoids, neoxanthin, crocin and $\beta$-carotene, assayed using protoplast co-culture method with digital image analysis. Plant Biotechnol., 38, 101-107. http://doi.org/10.5511/ plantbiotechnology.20.1211a

Seyama, T., Kimura, S., Sasamoto, H., Abe, H., \& Kondo, T. (2008). Spinning of a gigantic bundle of hollow fibrils by a spirally moving higher plant protoplast. Planta, 227, 1187-1197. http://dx.doi.org/10.1007/s00425-008-0689-1

Sheue, C. R., Liu, H. Y., \& Yong, J. W. H. (2003). Kandelia obovata (Rhizophoraceae), a new mangrove species from Eastern Asia. Taxon, 52, 287-294. https://doi.org/10.2307/3647398

Spalding, M., Kainuma, M., \& Collins, L. (2010). World atlas of mangroves. Earthscan, London. https://doi.org/10.4324/9781849776608

Suwa, R. (2014). Latitudinal gradient in forest structure and productivity in mangroves: The Ryukyu archipelago as the northern limit of mangrove distribution. Jpn. J. Int. Forest and Forestry, 91, 36-41. https://doi.org/10.32205/jjjiff.91.0_36

Suzuki, S., Wasano, N., Kimura, M., Yasuda, R., Nakagawa, W., Sasamoto, Y., Fujii, Y., \& Sasamoto, H. (2018). Evaluation of putative allelochemicals, cinnamic acid and an anthocyanin, in Spiraea thunbergii and $S$. cantoniensis using in vitro bioassay method of allelopathy, the protoplast co-culture with digital image analysis. Proceedings of the 4th International Conference of Asian Allelopathy Society. p. 33-72.

Tomlinson, P. B. (1986). The Botany of Mangroves. Cambridge University Press, New York.

Tsuchiya, S., Ogita, S., Kawana, Y., Oyanagi, T., Hasegawa, A., \& Sasamoto, H. (2013). Relation between amino acids profiles and recalcitrancy of cell growth or salt tolerance in tissue and protoplast cultures of three mangrove species, Avicennia alba, Bruguiera sexangula, and Sonneratia alba. Am. J. Plant Sci., 4, 1366-1374. https://doi.org/10.4236/ajps.2013.47167

Wakita, Y., Sasamoto, H., Yokota, S., \& Yoshizawa, N. (1996). Plantlet regeneration from mesophyll protoplasts of Betula platyphylla var. japonica. Plant Cell Rep., 16, 50-53. https://doi.org/10.1007/BF01275448

Wakita, Y., Yokota, S., Yoshizawa, N., Katsuki, T., Nishiyama, Y., Yokoyama, T., Fukui, M., \& Sasamoto, H. (2005). Interfamilial cell fusion among leaf protoplasts of Populus alba, Betula platyphylla and Alnus firma: assessment of electric treatment and in vitro culture conditions. Plant Cell Tiss. Org. Cult., 83, 319-326. 
https://doi.org/10.1007/s11240-005-8428-9

Yokota, S., Miki, T., Yoshizawa, N., \& Sasamoto, H. (2005). Isolation and Culture of Protoplasts from Cell Suspension Cultures of Prunus yedoensis. Bull. Coll. Agric Utsunomiya Univ., 19, 1-19.

\section{Copyrights}

Copyright for this article is retained by the author(s), with first publication rights granted to the journal.

This is an open-access article distributed under the terms and conditions of the Creative Commons Attribution license (http://creativecommons.org/licenses/by/3.0/). 